\title{
An overview of the in-vessel ICRF-diagnostics in the ASDEX Upgrade tokamak
}

\author{
H. Faugel ${ }^{1}$, R. Ochoukov ${ }^{1}$, V. Bobkov ${ }^{1}$, H. Fünfgelder ${ }^{1}$, J. M. Noterdaeme ${ }^{1,2}$, D. Aguiam $^{3}$, R. Dux ${ }^{1}$, \\ ASDEX Upgrade Team ${ }^{4}$ and EUROFUSION MST1 Teams ${ }^{5}$ \\ ${ }^{1}$ Max-Planck-Institut für Plasmaphysik, EURATOM Association, Garching, Germany \\ 2 EESA Department, Ghent Univerity, Belgium \\ ${ }^{3}$ Instituto de Plasmas e Fusão Nuclear, Lisboa, Portugal \\ ${ }^{4}$ See the author list A. Kallenbach et al, Nucl Fus 57 (2017) 102015 \\ 5 See the author list H. Meyer et al, Nucl Fus 57 (2017) 102014
}

Radio Frequency (RF) waves in the Ion Cyclotron Range of Frequency (ICRF) are successfully used to heat fusion plasmas. For a better understanding of the ICRF wave propagation, absorption and other processes in the plasma, the ICRF-related in-vessel diagnostics has been extended recently in the ASDEX Upgrade tokamak.

A number of high frequency B-field probes have been installed which, arranged as an array, pick up the magnetic field components of the radiated wave, and can thus be used to characterize the radiation spectrum of the ICRF-antennas during plasma discharges. Dual channel logarithmic RF-detectors with phase detection capability have been designed and installed to track the fast variations of the probe signals during events like edge localized modes (ELMs). The same probes are also used to measure the ion-cyclotron emission (ICE) of plasmas. A fast, open source data acquisition system, which digitizes the RF-signal with $125 \mathrm{MSamples} / \mathrm{s}$ and 14 bit amplitude resolution, records the ICE signals. To allow ICE measurements during ICRF operation notch filters are used to suppress the ICRF signal by $50 \mathrm{~dB}$. In order to characterize drive mechanisms and consequences of RF sheaths on the ICRF-antenna structures, 12 antenna limiter tiles are equipped with shunts to allow the measurements of RF- and DC-currents during operation, with some of the locations also covered by the spectroscopic lines of sight measuring the emission of neutral tungsten at $400.9 \mathrm{~nm}$ (WI). The antenna-embedded X-mode reflectometry with 3 channels operating simultaneously in the extended U-band helps to study ICRF wave coupling, whereas the RF current probes in the 3-strap ICRF antennas provide information on the phasing between central and outer straps.

Keywords: plasma diagnostics, ICRH, ICRF, ICE, radio frequency, reflectometer

\section{Introduction}

Radio frequency waves in the ion cyclotron range of frequencies (ICRF) are successfully used to heat magnetically confined plasmas to fusion relevant temperatures. This paper reviews the coverage of the ASDEX Upgrade vessel by the diagnostics relevant to characterize ICRF wave coupling and propagation, ion cyclotron emission, as well as Radio Frequency (RF) induced issues like sheath effects at antenna limiters. Bdot probes pick up the fast and slow waves along a magnetic field line, allowing to determine the radiation spectrum of an ICRF-antenna. The B-dot probes can also be used to detect Ion Cyclotron Emission (ICE) from the plasma. Insulated limiter shunts allow the DC current flow on limiter tile caused by sheath rectification in front of the antenna. Coaxial cables attached near the grounding point of the antenna loops are used to feedback control for a minimum of limiter surface sputtering. Microwave reflectometers allow the measurement of the density profile in front of the ICRF antenna. Eleven spectroscopy channels are observing tungsten coated tiles of one antenna to characterize the tungsten erosion. All these measurements provide more information to study the physics of the ICRF antennas and of the waves launched.

\subsection{Designing the B-dot probes}

The B-dot probes consist of a coil that is placed in a cover with a small slit at the front where the magnetic field can enter. As the probes have to be installed in the vacuum vessel, the cables and the coils have to withstand the baking at $150{ }^{\circ} \mathrm{C}$ for several days. The coil has to be coreless to prevent effects caused by a saturated ferrite core. Both, cables and coils have to be qualified to meet the requirements for ASDEX Upgrade in vessel installation. A triaxial cable with PTFE dielectric and an outer FEP jacket (elspec TR31601) was used connecting the ceramic RF coil (Sumida Series 5120, $177 \mathrm{nH}$ ). The probe case was made out of stainless steel and the $1 \times 10$ $\mathrm{mm}$ slit of the probe was covered with a $1 \mathrm{~mm}$ thin sheet of alumina to protect the coil from plasma. The size of the probe is $22 \times 22 \times 60 \mathrm{~mm}$, which is small enough to mount them in the quite crowded interior of ASDEX Upgrade vessel, e.g. some probes were mounted on top or underneath other existing diagnostics. All probes were mounted in a Spinner RG 100-230 measurement transmission line for calibration. A Rohde \& Schwarz vector network analyzer (VNA) was used to record the transfer function from 20 to $120 \mathrm{MHz}$. 


\subsection{B-dot probes during ICRF operation}

For measuring the radiation spectrum of one of the newer 3-strap antennas $[1,2]$ an array of 12 B-dot probes that are magnetically connected with the antenna were installed. The probes are mounted in pairs (see Fig. 1), with one probe oriented along the external magnetic field and the other oriented perpendicularly, thus enabling the characterization of the propagation of the fast and slow wave. The inclination of the probes was calculated for a standard discharge with $2.5 \mathrm{~T}$ magnetic field and $1 \mathrm{MA}$ plasma current. Two-channel phase detectors measure the phase differences between individual probes [3]. Amplitude and phase signals are digitized at a rate of $200 \mathrm{kHz}$ [4]. Beside the array a number of probe pairs are mounted on different positions, e.g. at the high field side on the opposite side of the four antennas or near the divertor. It is also possible to connect probes to a fast two-channel data acquisition system [5] that is equipped with two selectable notch filters to suppress the frequency at which ICRF is applied to the plasma. The center frequency of the notch filters is 30 and $36.5 \mathrm{MHz}$, the bandwidth of the notch is $1.4 \mathrm{MHz}$ with a maximum attenuation of $50 \mathrm{~dB}$. The data acquisition system [6] digitizes $1 \mathrm{~ms}$ long time slices at $125 \mathrm{MHz}$ sampling rate with 14 bit resolution followed by a $9 \mathrm{~ms}$ pause. This interleaving is caused by the limited memory of the data acquisition (512 MByte RAM), on the other hand a $1 \mathrm{~ms}$ long time slice can still fully cover fast plasma events like ELMs by chance. The acquired signals can be postprocessed into a series of spectra showing the time evolution of the discharge.

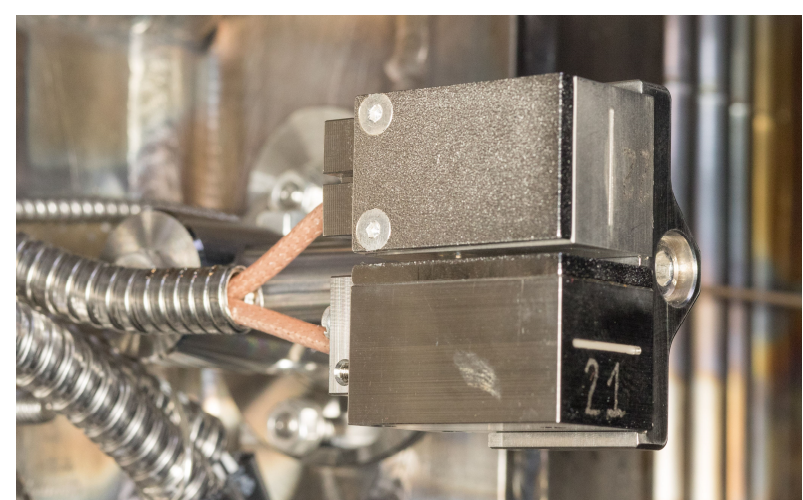

Fig. 1. Example of a B-dot probe pair installed at the highfield side in ASDEX Upgrade. The white vertical mark on the top probe and the horizontal mark on the bottom probe are the ceramic covered entrance slits of the probes. The front of the probe is shielded by tungsten coated graphite tiles (not shown) with slits of the probes aligned with the gaps between the tiles.

\subsection{B-dot probes without ICRF operation}

During discharge phases without ICRF it is possible to record ICE generated purely by the confined fast particles, as Fig. 2 demonstrates. As the magnetic field of a tokamak is a known function of the radial position and as the ratio of mass over charge is different for different ions, it is possible to locate the position of ICE. Together with other diagnostics it is possible to gain a better understanding on the mechanisms leading to ICE [7].

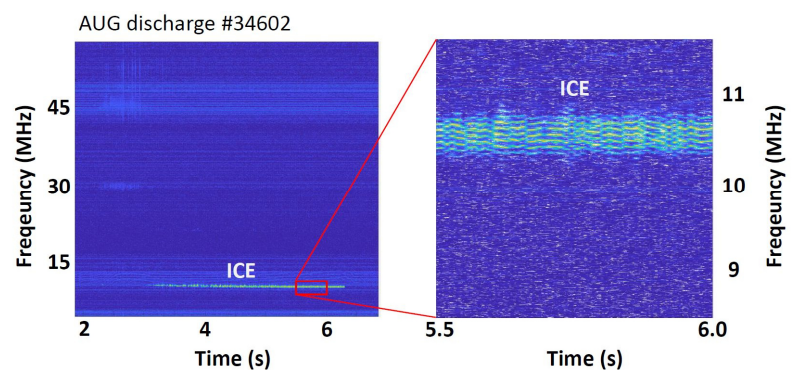

Fig. 2. Example of ion cyclotron emission (ICE) during a plasma discharge. The magnetic field was $2.5 \mathrm{~T}$ on the axis, the emission was likely caused by fusion-born tritium, note the frequency splitting.

\section{Limiter shunts}

As sheath rectification near ICRF antennas has been identified as the main source of impurity production during ICRF heating [2], a number of side limiter tiles have been installed with measurements of DC and RF currents that are picked up. The limiter tiles are electrically connected to the limiter support frame with a shunt made out of Inconel (Fig. 3). The shunts were calibrated after installation with a DC-current source. The resistance of the shunt is $3 \mathrm{mOhm}$, the calculated inductivity is $33 \mathrm{nH}$ results in a $3 \mathrm{~dB}$ bandwidth from DC to $15 \mathrm{kHz}$, the $240 \mathrm{pF}$ parallel capacitance can be neglected as well as the skin effect in this frequency range. The measurement signal is fed into a coaxial cable. As coaxial cable can also transmit RF signals with low attenuation, it was decided to split up the shunt signals into a DC and a RF component. The RF signal is extracted with a $2 \mathrm{MHz}$ high-pass filter and fed into an $\mathrm{RF}$ detector with a logarithmic response, the detection range spans from $-45 \mathrm{dBm}$ to $+23 \mathrm{dBm}$ into $50 \mathrm{Ohm}$. For a measurement of the transfer characteristic (S21) the limiter shunt assembly was connected to port 1 of a vector network analyzer with a thin semi-rigid coax cable; port 2 was connected to the coax cable of the shunt. The result is shown in Fig. 4. This data was used to derive an equivalent circuit consisting of a set of microstrip and coaxial transmission lines, but because of the complex geometry of the limiter tile and the shunt only a rough approximation was possible. Simulations with 3D-electromagnetic (EM) simulation software will be used in the near future for modelling. Further measurements of the limiter shunt assembly included the sensitivity to mutual coupling using a magnetic loop antenna (Siemens STRA 3885). The measurements of S21 between the loop antenna and the shunt showed that the sensitivity of the slow wave is constant over a frequency range from 5 to $30 \mathrm{MHz}$ (frequency limit of the loop antenna) at $-68 \mathrm{~dB}(+/-1 \mathrm{~dB})$; the sensitivity of the fast wave increased form $-84 \mathrm{~dB}(+/-2 \mathrm{~dB})$ at 5 $\mathrm{MHz}$ to $-75 \mathrm{~dB}(+/-2 \mathrm{~dB})$ at $30 \mathrm{MHz}$. As the newer 3strap antenna allows the independent control of power ratio and phase between the central strap and the outer 
straps, the direct influence of these parameters on the impurity production could be shown.

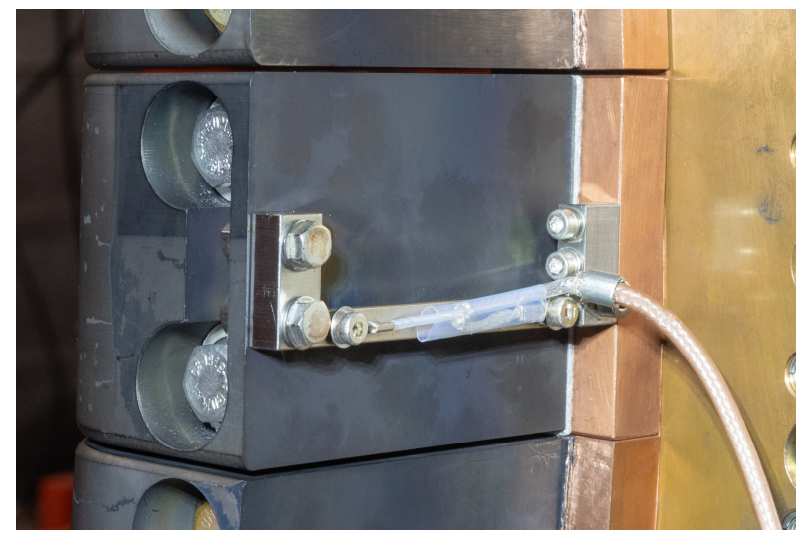

Fig. 3. The shunt is mounted on the backside of a limiter tile and insulated in such a way, that electrical currents have to pass through it. A coaxial cable transmits the current signal to the data acquisition system.

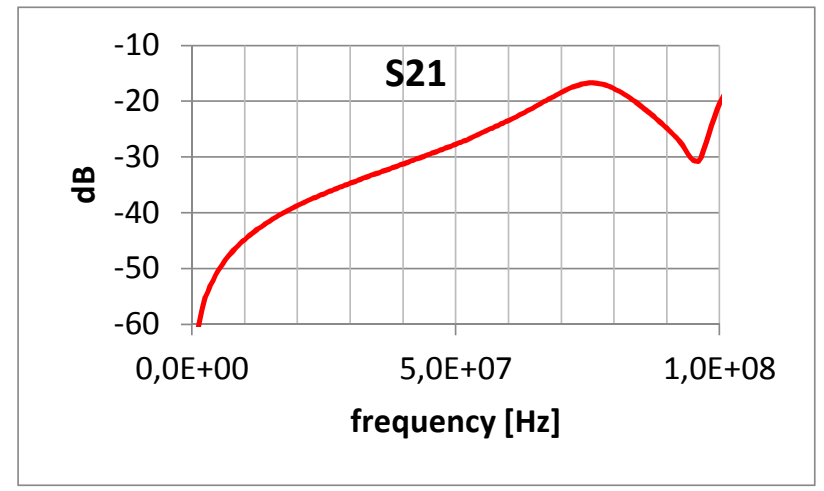

Fig. 4. Transfer function S21 measured with a vector network analyzer (VNA) of the limiter shunt assembly.

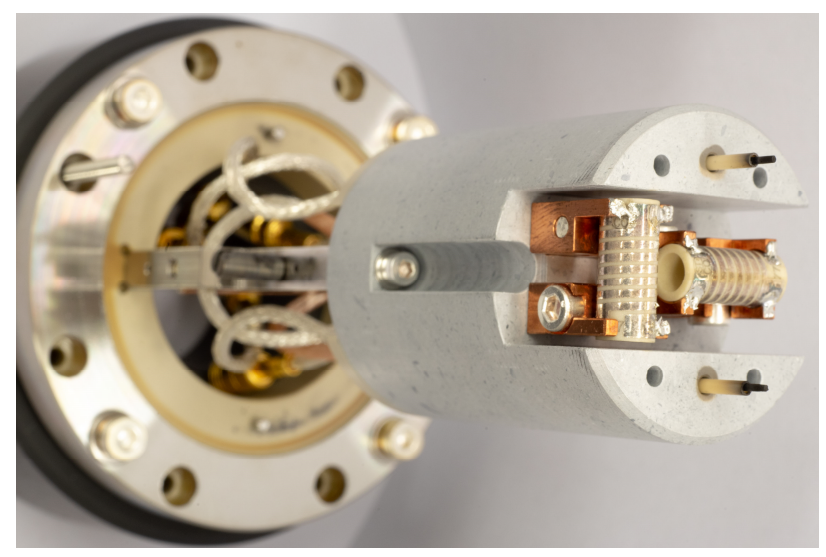

Fig. 5. A view of the manipulator head. The cover was removed to show the two pick up coils and the two Langmuir probes (black tips).

\section{ICRF manipulator head}

ASDEX Upgrade is equipped with a fast moving midplane manipulator that allows measurements inside the plasma. For this purpose a probe head with two Langmuir probe and two B-dot probes was designed, as shown in Fig. 5. The cover of this probe head is made of tungsten-coated graphite. The manipulator head has been successfully used during the last ASDEX-Upgrade campaign, with up to 14 strokes during a discharge. One stroke takes $0.25 \mathrm{~s}$, leaving the probe for about $0.1 \mathrm{~s} 10$ $\mathrm{mm}$ deep in the plasma. The results of this diagnostic will be part of a $\mathrm{PhD}$ study.

\section{Antenna probes}

To operate the 3-strap antennas in the best possible way, it is necessary to control the power ratio of the central and outer straps and the phase between them. Whereas the power ratio can be controlled either with appropriate settings of the RF-generators one needs an active feedback control of the phase between antenna straps. As the electrical length of the antenna feeding transmission lines is changing as a matter of the standing wave pattern at the ceramic supports, we opted for a measurement of the current at the grounding points of the antenna loops. The voltage drop along a small, several millimeter long path at the end of the antenna loops (see Fig. 6) gives a signal that is proportional to the current in the antenna loop. The signal is connected by a thin semi-rigid coaxcable (UT-85). While the absolute calibration of such current probes is not straightforward, they provide accurate information about phase between antenna straps, necessary for the phase controller of the RF generators.

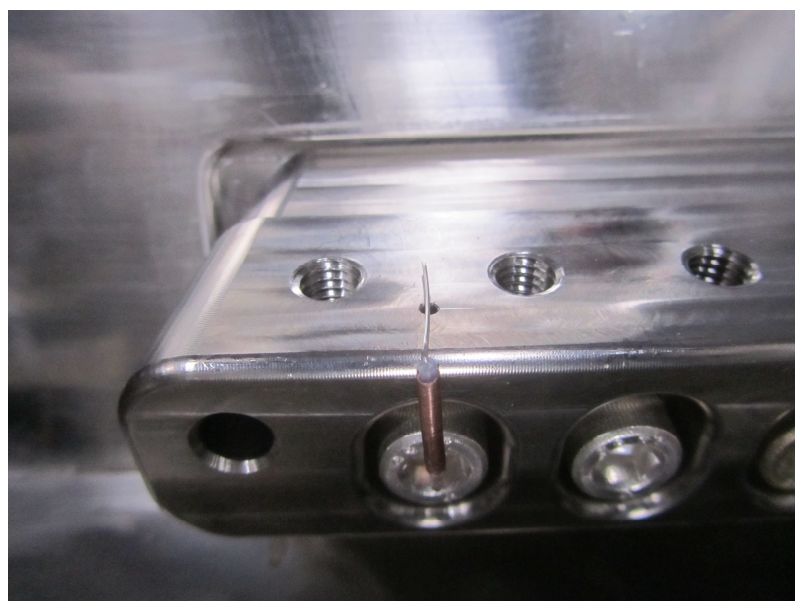

Fig. 6. The thin semi-rigid coax cable during installation in the antenna loop support. The outer connector will be connected to the support, whereas the inner conductor will be fixed in a small hole drilled in the antenna loop (not installed yet).

\section{Microwave reflectometers}

During the installation of the new three-strap antennas in total 20 microwave antennas have been integrated, allowing operation of microwave reflectometers at ten different positions in the antenna or near the antenna limiters. One reflectometer antenna pair close to the ICRF antenna limiter is shown in Fig. 7. Due to limitations of the port diameter, only seven out of ten antenna pairs can be connected to the outside of the vessel. The current setup of the microwave transmitters and receivers allows operating three antenna pairs in Xmode using the extended U-band from 40 to $68 \mathrm{GHz}$. The sweep time is $15 \mu \mathrm{s}$. The system can measure density profiles up to $2 \times 10^{19} \mathrm{~m}^{-3}$. An update to run six 
antenna pairs is under discussion. The system is described in [8] and results can be found in [9].

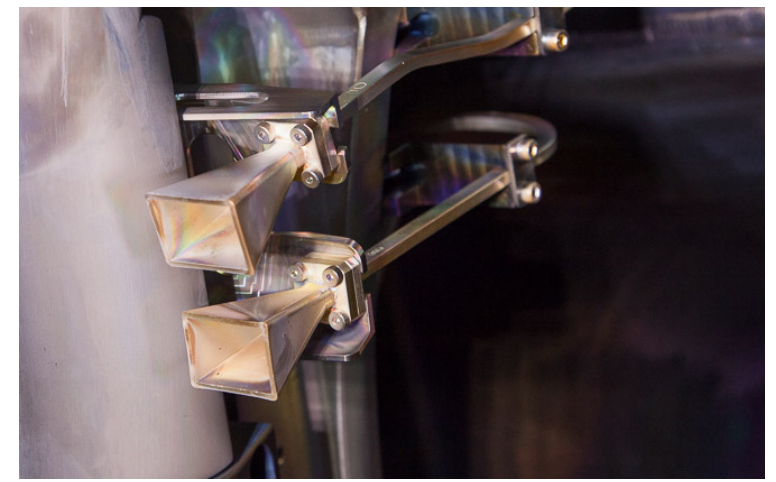

Fig. 7. A view showing a reflectometer antenna pair near the ICRF antenna limiters.

\section{Limiter spectroscopy}

With the introduction of the new 3-strap antennas, eleven lines of sight (LOS) of the limiter spectroscopy diagnostics were re-aligned to look at the tungsten covered surface of the limiter tiles of the 3-strap antennas and measure the WI spectral line emission [10]. Experiments with scans in power ratio and phase between the central and outer straps of the antenna show a good correlation of the intensity of tungsten line emission (and thus the tungsten influx) and limiter current measurements [11].
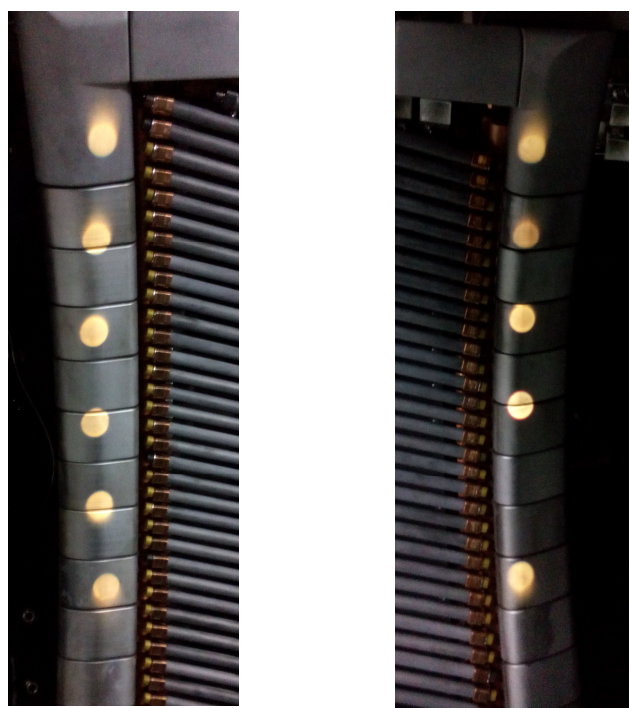

Fig. 8. Left: Photographs of the spectroscopic LOSs highlighted on the left side of the 3-strap ICRF antenna. Right: LOSs at the right side of the antenna.

\section{Conclusions}

Interactions between plasma and ICRF power for plasma heating involves complex processes and its understanding is steadily improving. The installation and operation of different probes in addition to the spectroscopic diagnostics provides valuable data to characterize the effects like ICRF wave propagation and
RF sheath rectification. Operation of the microwave reflectometers gives detailed information about the plasma density in front of ICRF antennas which helps further modelling and optimizing the ICRF antennas and of the coupling of the ICRF power to the plasma.

\section{Acknowledgments}

This work has been carried out within the framework of the EUROfusion Consortium and has received funding from the Euratom research and training programme 2014-2018 under grant agreement No 633053. The views and opinions expressed herein do not necessarily reflect those of the European Commission.

The authors want to thank Gerhard Siegl and Bertram Brucker for designing the B-dot probes and the supports for the probes. Many thanks also to the ASDEX Upgrade diagnostic mechanic team for their help during installation of the different diagnostics.

\section{References}

[1] H. Fünfgelder et al., A double success story: The international cooperation to build the new ICRF antennas on ASDEX Upgrade and the results obtained, Fus. Eng. Des. 123 (2017) 40-46.

[2] V Bobkov et al., Making ICRF power compatible with a high-Z wall in ASDEX Upgrade, Plasma Phys. Control. Fusion 59 (2017) 014022.

[3] A. Mlynek, A simple and versatile phase detector for heterodyne interferometers, Rev. Sci. Instrum., 023504 (2017).

[4] K. Behler et al., Deployment and future prospects of high performance diagnostics featuring serial I/O (SIO) data acquisition (DAQ) at ASDEX Upgrade Fusion Engineering and Design 87 (2012) 2145-2151.

[5] R. Ochoukov et al., Major upgrades of the high frequency B-dot probe diagnostic suite on ASDEX Upgrade, EPJ Web of Conferences 157, 03038 (2017).

[6] www.redpitya.com

[7] R. Ochoukov, to be submitted to Nuclear Fusion.

[8] D.E. Aguiam, et al., Implementation of the new multichannel X-mode edge density profile reflectometer for the ICRF antenna on ASDEX Upgrade, Rev. Sci. Instrum., 87 (11) (2016), pp. 3-5.

[9] D.E. Aguiam, Implementation of a X-Mode multichannel edge density profile reflectometer for the new ICRH antenna on Asdex Upgrade, PhD thesis, Universidade de Lisboa, Ghent University

[10] V. Bobkov et al., Progress in controlling ICRF-edge interactions in ASDEX upgrade, AIP Conference Proceedings 1689, 2015.

[11] V. Bobkov, Characterization of 3-strap antennas in ASDEX Upgrade, EPJ Web of Conferences 157, 03005 (2017). 